\title{
The influence of emotional distraction on verbal working memory: An fMRI investigation comparing individuals with schizophrenia and healthy adults
}

\author{
Michele T. Diaz ${ }^{1,4}$, George He $\mathrm{He}^{2,4}$, Syam Gadde ${ }^{1,4}$, Carolyn Bellion ${ }^{3}$, Aysenil Belger ${ }^{1,3,4}$, \\ James T. Voyvodic ${ }^{1,4}$, and Gregory McCarthy ${ }^{2,4}$ \\ ${ }^{1}$ Brain Imaging and Analysis Center, Duke University, Durham NC \\ 2 Psychology Department, Yale University, New Haven, CT \\ ${ }^{3}$ Department of Psychiatry, University of North Carolina, Chapel Hill, NC \\ 4 The Biomedical Informatics Research Network
}

\section{Abstract}

The ability to maintain information over short periods of time (i.e., working memory) is critically important in a variety of cognitive functions including language, planning, and decision-making. Recent functional Magnetic Resonance Imaging (fMRI) research with healthy adults has shown that brain activations evoked during the delay interval of working memory tasks can be reduced by the presentation of distracting emotional events, suggesting that emotional events may take working-memory processes momentarily offline. Both executive function and emotional processing are disrupted in schizophrenia, and here we sought to elucidate the effect of emotional distraction upon brain activity in schizophrenic and healthy adults performing a verbal working memory task. During the delay period between the memoranda and memory probe items, emotional and neutral distractors differentially influenced brain activity in these groups. In healthy adults, the hemodynamic response from posterior cingulate, orbital frontal cortex, and the parietal lobe strongly differentiated emotional from neutral distractors. In striking contrast, schizophrenic adults showed no significant differences in brain activation when processing emotional and neutral distractors. Moreover, the influence of emotional distractors extended into the memory probe period in healthy, but not schizophrenic, adults. The results suggest that although emotional items are highly salient for healthy adults, emotional items are no more distracting than neutral ones to individuals with schizophrenia.

\section{Keywords}

Working Memory; Emotion; Schizophrenia; fMRI

(C) 2011 Elsevier Ltd. All rights reserved.

Corresponding Author: Michele T. Diaz, Brain Imaging and Analysis Center, Duke University, 2424 Erwin Rd, Suite 501, Durham, NC 27705 p: 919-681-9528 f: 919-681-7033, mtd3@duke.edu.

Conflict of Interest Statement: The authors have no financial, personal or other relationships with other people or organizations within three (3) years of beginning the work submitted that could inappropriately influence, or be perceived to influence, this work.

Publisher's Disclaimer: This is a PDF file of an unedited manuscript that has been accepted for publication. As a service to our customers we are providing this early version of the manuscript. The manuscript will undergo copyediting, typesetting, and review of the resulting proof before it is published in its final citable form. Please note that during the production process errorsmaybe discovered which could affect the content, and all legal disclaimers that apply to the journal pertain. 


\section{INTRODUCTION}

Schizophrenia is a disorder characterized by disruptions in perception. Individuals with schizophrenia may perceive stimuli that are not present (e.g., auditory or visual hallucinations) or may respond to stimuli in an uncharacteristic manner (e.g., lack of affective response to emotional stimuli). In addition, schizophrenia can profoundly impair cognitive functioning. Some have suggested that a primary deficit in working memory may underlie many of the cognitive deficits seen in schizophrenia (Goldman-Rakic, 1994). For example, verbal dysfluencies (Conklin, Curtis, Katsanis, \& Iacono, 2000; Heinrichs \& Zakzanis, 1998) may persist due to an inability to maintain an ordered progression of thoughts or words. A focus on external stimuli may indicate a lack of ability to maintain an internal list of thoughts or goals (Goldman-Rakic, 1994).

Working memory, the ability to maintain and manipulate information over brief periods of time, is vital to many cognitive processes including language, planning, and decision making. Verbal working memory, working memory in sentences, words, and letters, relies heavily on prefrontal cortices (Cohen et al., 1994; Cohen et al., 1997; McCarthy et al., 1994; Petrides, Alivisatos, Meyer, \& Evans, 1993). Middle frontal gyrus has been shown to be engaged by several working memory tasks including spatial working memory (McCarthy et al., 1994), number generation and number memory (Petrides et al., 1993), and letter memory (Sweet et al., 2008). Activation in inferior frontal gyrus (IFG) has been observed during a variety of linguistic tasks such as memory for letters (Cohen et al., 1997), semantic categorization (Kapur et al., 1994; Roskies, Fiez, Balota, Raichle, \& Petersen, 2001), semantic association (Noppenney, Phillips, \& Price, 2004), semantic priming (Copland, De Zubicaray, McMahon, \& Eastburn, 2007; Demb et al., 1995; Gold et al., 2006), rhyme judgments (Paulesu, Frith, \& Frackowiak, 1993; Roskies et al., 2001), pseudo-homophone naming (Owen, Borowsky, \& Sarty, 2004), and phoneme monitoring (Demonet et al., 1992; Zatorre, Evans, Meyer, \& Gjedde, 1996).

The neural infrastructure of working memory is of considerable relevance to clinical disorders in which executive function is impaired, such as schizophrenia. Several studies suggest that individuals with schizophrenia elicit less brain activation compared to healthy adults during working memory tasks. Using a two-back task with Korean alphabetical letters, individuals with schizophrenia elicited decreased activation in inferior frontal, middle frontal, and superior temporal gyri compared to healthy controls (Pae et al., 2008). Similarly, during an N-back task using English letters patients elicited less activation in right dorsolateral prefrontal cortex (DLPFC) than healthy adults (Perlstein, Dixit, Carter, Noll, \& Cohen, 2003). Decreased activation in DLPFC for patients compared to healthy controls has also been observed during continuous performance tasks (Barch et al., 2001; Perlstein et al., 2003). Children of schizophrenic individuals (high risk offspring) have also been found to elicit less activation in DLPFC and inferior parietal cortex compared to healthy controls during a spatial working memory task (Keshavan et al., 2002).

In addition to differences in levels of activation, the extent of activation may be influenced by task difficulty. At lower difficulty levels (e.g., 0-back, 1-back) activation was similar among patients and controls, but at more difficult loads, activation continued to increase linearly for healthy adults, but declined in patients (Perlstein, Carter, Noll, \& Cohen, 2001). Moreover, they found that load-dependent increases in prefrontal activation were negatively correlated with the severity of disorganized symptoms in the patients. In a separate study, although load-dependent increases in activation were found for all participants, the magnitude of the increases were significantly smaller in patients compared with healthy controls (Perlstein et al., 2003). 
Beyond DLPFC, others have reported reduced activation for adults with schizophrenia. During a spatial working memory task, although there were no differences in fMRI activation in DLPFC, patients elicited less activation in left anterior cingulate and bilateral parietal cortex compared to healthy controls (Kindermann, Brown, Zorrilla, Olsen, \& Jeste, 2004). Similarly, Schneider and colleagues found that patients elicited decreased activation in the precuneus during an N-back task compared to healthy controls, although they also reported increased activation in ventrolateral prefrontal cortex (Schneider et al., 2007).

However, others have reported no significant differences in brain activation comparing individuals with schizophrenia and control participants. Honey and colleagues (Honey, Bullmore, \& Sharma, 2002) found that both patients and healthy controls strongly activated the frontal-parietal network during a verbal N-back task, with no significant differences between groups. Interestingly, activation in posterior parietal cortex was positively correlated with reaction time in healthy adults, but not in patients.

There have been several reports of differences in brain activation between patients and controls that indicate increases in activation in dorsolateral prefrontal cortex (DLPFC) for patients (Manoach et al., 2000; Manoach et al., 1999; Potkin et al., 2009). In a recent study, both groups showed significant activation in DLPFC during a Sternberg Item Recognition Paradigm (SIRP), however, patients elicited significantly greater activation than healthy controls (Potkin et al., 2009). Moreover, the pattern of increased activation remained when performance was matched across a subset of participants from the two groups. The authors characterized these changes as inefficiencies in processing because the increased activation did not correspond to performance improvements. Van Raalten and colleagues examined the role of familiarity in working memory processes by comparing activation during the SIRP with practiced and novel stimuli. With novel stimuli, patients elicited larger patterns of activation in left prefrontal regions than healthy controls. Although both groups showed decreases in activation with practiced stimuli, only in healthy controls did the magnitude of this decrease correspond to improvements in performance (van Raalten, Ramsey, Jansma, Jager, \& Kahn, 2008). Increased brain activation was also found among first-degree relatives of individuals with schizophrenia during a verbal auditory continuous performance task (Thermenos et al., 2004). Relatives elicited greater task-related activation in prefrontal regions, as well as in thalamus and anterior cingulate compared to healthy adults. These findings suggest that neurocognitive dysfunction associated with schizophrenia extends across a variety of cognitive tasks and may extend to close relatives. Additionally, the behavioral (Honey et al., 2002) and functional differences in individuals with first-episode schizophrenia (Barch et al., 2001; Perlstein et al., 2001; Schneider et al., 2007), and in relatives (Keshavan et al., 2002; Thermenos et al., 2004) suggest that neurocognitive changes may be at least partially independent of symptom duration. While these studies have examined executive function in patient and control participants, they have not examined the interaction of emotional processing and executive function in individuals with schizophrenia.

Previous research has suggested that different networks are involved in executive function and emotional processing. Several lines of research have proposed a dorsal-ventral distinction such that a dorsal network including middle frontal gyrus, parietal cortex, and posterior cingulate is involved in executive tasks, while ventral brain regions such as inferior frontal gyrus, orbital frontal cortex, and the amygdala are involved in emotional processing (Anticevic et al., 2010; Fichtenholtz et al., 2004; Phan, Wager, Taylor, \& Liberzon, 2002; Phan, Wager, Taylor, \& Liberzon, 2004; Phelps \& Ledoux, 2005; Wang, Labar, \& McCarthy, 2006; Wang, McCarthy, Song, \& Labar, 2005; Yamaskai, Labar, \& McCarthy, 2002). Others have suggested that the insula, in particular anterior insular cortex, is involved 
in interoceptive and emotional sensation, and that the insula along with anterior cingulate cortex may underlie consciousness (Craig, 2002; 2009).

More recently, research has probed how emotion and distraction interact in working memory among individuals with schizophrenia. Dichter and colleagues investigated the role of emotional and neutral distractors during a visual oddball task in individuals with schizophrenia and healthy control participants (Dichter, Bellion, Casp, \& Belger, 2009). Consistent with previous results, healthy control participants elicited activation in dorsolateral prefrontal regions to target stimuli, while emotional stimuli elicited activation in ventral frontal brain regions. However, individuals with schizophrenia elicited less activation to target stimuli in dorsolateral prefrontal regions and less activation to emotional distractors in ventral-frontal brain regions. In addition, the schizophrenic patients also showed less deactivation within these brain regions when compared to healthy adults. These results suggest that brain regions involved in both emotional processing and executive function may be dysfunctional in schizophrenia.

In the present study, we utilized a verbal working memory task that incorporated emotional and neutral distractors. Participants were instructed to remember a series of eight words that were followed by a delay period that contained either emotional or neutral distracting photographs (International Affective Picture System: IAPS). Following the delay period, participants were shown eight pairs of words and asked to decide which word in each pair was presented in the initial memoranda. Of greatest interest is the comparison between emotional and neutral trials in patients and controls. We hypothesized that healthy adults, would show greater activation to emotional distractors compared to neutral distractors in ventral brain regions, such as amygdala, and orbital frontal cortex. Moreover, we predicted that patients would not differentiate emotional and neutral distractors as strongly as healthy adults. With respect to the executive network, previous research suggests that patients inefficiently activate the frontal-parietal network, as evidenced by reduced activations (Barch et al., 2001; Dichter et al., 2009; Pae et al., 2008; Perlstein et al., 2001; Perlstein et al., 2003), and activations that are unrelated to performance measures (Honey et al., 2002; van Raalten et al., 2008). Based on those results we expected that patients would show reduced patterns of activation in the executive frontal-parietal network compared to healthy adults, and that these patterns of activation would not be strongly related to behavioral performance.

\section{METHODS}

\section{Participants}

Seventeen healthy young adults (Healthy Controls) and eleven adult patients with schizophrenia participated in this study. Healthy controls had no history of neurological or psychological disorders and had an average age of 24.01 (age range 19-30; 7 male; all right handed). Schizophrenic patients had an average age of 32.57 (age range 20 - 59; 10 male; 8 right handed). The patient group had an average illness duration of 13.7 years (illness duration range $4-50$ years, $\mathrm{SD}=14.52$ ). All patients were being treated with antipsychotic medication at the time of study. In addition to antipsychotic medication, 6 patients were also being treated with anti-depressants, and two others were being treated with an antipsychotic and lithium. None of the patients were being treated with anticholinergics at the time of study. There was a significant difference in age between groups $(t=-2.24$, corrected $\mathrm{df}=11.18, \mathrm{p}<.05$ ), and in the number of males and females between the two groups $\left(\chi^{2}=6.925, \mathrm{df}=1, \mathrm{p}, .01\right)$. There were no significant differences between groups in handedness $\left(\chi^{2}=2.32, \mathrm{df}=3, \mathrm{p}=.509\right)$ or race $\left(\chi^{2}=1.258, \mathrm{df}=2, \mathrm{p}=.533\right)$. Additional demographic information on the patients is provided in Table 1. All participants were native English speakers and had normal or corrected to normal vision. Each participant provided informed 
consent and was paid for his or her participation. All experimental procedures were approved by the Duke University Medical Center Institutional Review Board.

\section{Stimuli}

Stimuli consisted of words, IAPS photographs (Lang, Bradley, \& Cuthbert, 2008), and scrambled images. Verbal stimuli were content words and were presented centrally. Across conditions words were equated for length (range: $3-12$ letters, mean length of words in trials with emotional distractors $=5.3$ letters; mean length of words in trials with neutral distractors $=5.4$ letters) and frequency (range: $1-472$, mean frequency of words in trials with emotional distractors: 47.9; mean frequency of words in trials with neutral distractors: 41.1)(Coltheart, 1981; Kucera \& Francis, 1967; Wilson, 1988). IAPS photographs were either neutral or negative. Negative IAPS photographs had a mean arousal rating of 5.44 (range $3.85-7.35$ ) and a mean pleasantness rating of 2.9 (range $1.78-4.50$ ). Neutral IAPS photographs had a mean arousal rating of 3.40 (range 1.76-4.23) and a mean pleasantness rating of 5.48 (range $4.03-6.91$ ) (Lang et al., 2008). Scrambled images were created by performing a Fourier transform on each IAPS photograph, permuting the phase spectrum, and then computing an inverse transform. The overall luminance of the resulting images was then adjusted to match the original photograph. This procedure ensured that the scrambled images retained the frequency spectrum of the original photographs, but were unrecognizable as scenes.

Ten healthy adult volunteers completed 8 task runs, and seven completed four runs (avg = 6.35). Eight patients with schizophrenia completed 8 runs, and 3 completed 4 runs (avg = 6.91). All stimuli were presented via Liquid Crystal Display (LCD) goggles (MRI Resonance Technologies, Los Angeles, CA, USA) using the CIGAL experimental control program (Voyvodic, 1999). Responses were made via a fiber optic button box (Current Designs, Philadelphia, PA, USA).

\section{Experimental Task}

Each trial of the blocked working memory task consisted of 3 segments (segment duration $=16 \mathrm{~s}$, trial duration $=48 \mathrm{~s}$ ): encoding word memoranda, maintaining the memoranda while viewing distractor photographs, and a forced-choice probe period. A depiction of the task is provided in Figure 1. Each segment consisted of 8 items, presented sequentially (item duration $=2 \mathrm{~s}$; segment duration $=16 \mathrm{~s}$ ). During the memoranda segment, words were presented in upper case and participants were instructed to remember these words. During the maintenance period distractors were either emotional or neutral IAPS photographs. The forced-choice probe period consisted of word pairs presented side by side and in lower case. For each word pair participants were asked to decide which of the two words was presented during the memoranda segment. The ordering of the items during the forced choice segment was randomized, and within a session foils and memoranda were not repeated. Trials were separated by a baseline period of unidentifiable, phase-scrambled images (item duration $=$ $2 \mathrm{~s}$; segment duration=16s). The two trial types (emotional and neutral) were identical in format except for the valence of the distractor photographs. Each run began and ended with the presentation of a fixation cross to allow for acquisition of a hemodynamic response baseline and offset (duration $=6 \mathrm{~s}$ ). Each run consisted of 4 trials, and the ordering of trials was randomized to minimize the participant's anticipation of the trial type. Participants received a practice session to ensure they could competently perform this task.

In order to ensure participants viewed the distractor photographs, and to assess the influence of an additional task, half of the participants were asked to complete a secondary face detection task during the distractor segment. During this segment, participants were instructed to press one button if they could see a human face and another button if they 
could not see a human face. The primary working memory task was identical for all participants.

\section{Acquisition of MRI Data}

MRI scanning was completed on a 3.0 Tesla GE EXCITE HD whole-body $60 \mathrm{~cm}$ bore human scanner equipped with $40 \mathrm{mT} / \mathrm{m}$ gradients and a $150 \mathrm{~T} / \mathrm{m} / \mathrm{s}$ slew rate. An eightchannel head coil was used for Radio Frequency reception (General Electric, Milwaukee Wisconsin, USA). Sagittal T-1 weighted localizer images were acquired and used to define a volume for high order shimming. The anterior and posterior commisures were identified for slice selection and shimming. A semi-automated high-order shimming program was used to ensure global field homogeneity. High-resolution structural images were acquired using a 3D fast Spoiled Gradient Recalled (fSPGR) pulse sequence (TR=7.356ms; TE $=2.988 \mathrm{~ms}$; FOV $=24 \mathrm{~cm}^{2}$; flip angle $=12^{\circ}$; voxel size $=.9375 \times .9375 \times 1.9 \mathrm{~mm}$; 68 contiguous slices $)$. Functional images sensitive to blood oxygen level-dependent (BOLD) contrast were acquired using an inverse spiral pulse sequence $\left(\mathrm{TR}=2.0 \mathrm{~s} ; \mathrm{TE}=27 \mathrm{~ms} ; \mathrm{FOV}=24 \mathrm{~cm}^{2}\right.$; flip angle $=60^{\circ}$; voxel size $=3.75 \times 3.75 \times 3.8 \mathrm{~mm} ; 34$ contiguous axial slices). Each of 8 runs consisted of the acquisition of a time series of 142 brain volumes. Three initial radio frequency $(\mathrm{RF})$ excitations were performed to achieve steady state equilibrium, and were subsequently discarded. The total length of each run was 4 minutes, 50 seconds.

\section{Data Analysis}

FSL was used to assess functional activations to each segment of the trial: scrambled baseline, memoranda, distractor, and forced-choice probe. Preprocessing and first level analyses of each individual run for each participant were performed using FSL version 4.1.0 [Oxford Centre for Functional Magnetic Resonance Imaging of the Brain (FMRIB), Oxford University, U.K.] (Smith et al., 2004; Woolrich et al., 2009). Functional image data were motion-corrected, high-pass filtered, and spatially smoothed using a Gaussian kernel (Full Width Half Maximum $=5 \mathrm{~mm}$ ). No participant had greater than $3 \mathrm{~mm}$ movement in the $\mathrm{X}, \mathrm{Y}$, or $\mathrm{Z}$ dimension. Pre-whitening or voxel-wise temporal autocorrelation was estimated and corrected using FMRIB's Improved Linear Model (FILM) (Woolrich, Ripley, Brady, \& Smith, 2001). The skull and other coverings were stripped from the structural brain images using the FSL brain extraction tool (Smith, 2002). Functional images of each participant were co-registered to structural images in native space, and structural images were normalized to the MNI standard brain supplied with FSL. The same transformation matrices used for structural-to-standard transformations were then used for functional-to-standard space transformations of co-registered functional images. A $\gamma$ function was used to model the hemodynamic response for each segment in each run. The FSL FIRST component was used for an individualized segmentation and labeling of subcortical anatomical regions. The Wake Forest University (WFU) Pick Atlas was used to segment cortical regions.

The first level analyses from the experimental runs of each participant were combined and a second level analysis was performed for each participant. These second level analyses were then combined according to group, into a group level analysis using the FMRIB Local Analysis of Mixed Effects (FLAME) (Beckman, Jenkinson, \& Smith, 2003; Woolrich, Behrens, Beckman, Jenkinson, \& Smith, 2004) to identify voxels that were activated by each trial type and segment. A fourth-level analysis was also completed to examine the data for interactions between participant group and trial type. Our main comparison of interest is the interaction between trial type (emotional and neutral) and participant group (healthy controls and patients).

In addition to the above, the single trial peri-event averages for each trial and segment were measured, and a time-point by time-point t statistic was calculated at each voxel (Gadde \& 
McCarthy, 2009). These t-statistic peri-event waveforms were combined across participants using a random effects analysis, and the difference in the mean amplitude of the hemodynamic response for each voxel was determined. Percent signal change was determined by averaging the hemodynamic response elicited by each trial type and segment and calculating the difference between baseline and peak points for each condition. Significance was thresholded at $\mathrm{p}<.01$ in these subsequent analyses. Coordinates of the centroids of activation and their corresponding anatomical gyri were determined through the use of anatomical atlases (Lancaster et al., 2000; Talairach \& Tournoux, 1988). All reported coordinates are in Montreal Neurological Institute (MNI) space. Results are displayed overlaid on an individual participant's brain normalized to MNI space.

\section{RESULTS}

\section{Behavioral Results}

We examined the response times and accuracies using two-way Analyses of Variance (ANOVAs) with group (healthy controls, patients) and trial type (neutral, emotional) as factors. For response times during the forced choice task, there was a main effect of group $(\mathrm{F}=8.66(1), \mathrm{p}<.005$; patients $=1130 \mathrm{~ms}$; healthy adults $=1010 \mathrm{~ms})$, but no main effect of trial type $(\mathrm{F}=.01(1), \mathrm{p}=.921)$, nor a group by trial type interaction $(\mathrm{F}=.001(1), \mathrm{p}=.973)$. For accuracies during the forced choice task, there was again a main effect of group $(\mathrm{F}=12.95$ (1), $\mathrm{p}<.001$; patients $=59.3 \%$, healthy controls $=79.5 \%$ ), but no main effect of trial type $(\mathrm{F}=.037(1), \mathrm{p}=.849)$, nor trial type by group interaction $(\mathrm{F}=.086(1), \mathrm{p}=.771)$.

We also examined the response times and accuracy information from the secondary facedetection task that half of the participants performed during the maintenance period to assess task-compliance. There was a significant main effect of group in the response time data $(\mathrm{F}=8.1(1), \mathrm{p}=.013$; patients $=956.10 \mathrm{~ms}$, healthy controls $=806.71 \mathrm{~ms})$, but no main effect of trial type $(\mathrm{F}=.018(1), \mathrm{p}=.894)$, nor group by trial type interaction $(\mathrm{F}=2.5(1), \mathrm{p}=.13)$. There were no differences in accuracy for any of the comparisons ( $\mathrm{F}=.009(3), \mathrm{p}=.999)$. Behavioral data from 3 patients was lost due to computer error.

\section{fMRI Results}

Encode Period-Both groups elicited activation in bilateral inferior frontal gyrus, which extended through the insula and precentral gyrus. Both groups also elicited activation in bilateral occipital and fusiform gyri, left parietal cortex, and a midline activation that extended into right and left medial frontal gyrus. There were no significant differences in brain activation between trial types or groups during this period, and there were no significant interactions between group and trial type (see Figure 2). Cluster coordinates from the overall activation during the Encode period are provided in Table 2. Results from the two groups during this period were collapsed into one Table because of the lack of differences between groups and trial types. Insert Figure 2 and Table 2 about here.

Maintenance (Distractor) Period-The primary comparisons examined the difference between Emotional and Neutral trials for each participant group, and the interaction between trial type and participant group. During the maintenance period, healthy controls showed extensive patterns of activation. Significant differences between Emotional and Neutral trials in healthy adults were found in bilateral occipital and fusiform gyri (Right: 50, -72, 4; Left: $-48,-78,12$ ), bilateral orbital and medial frontal cortices (Right: 4, 38, -20; Left: -4 , $38,-22$ ), bilateral cerebellum (Right: 16, -74, -32; Left: $-14,-72,-32$ ), left middle temporal gyrus $(-48,-16,-16)$, and bilateral middle frontal and precentral gyri (Right: 40 , 8, 26; Left: $-42,12,20)$. Schizophrenic patients elicited a larger response to emotional trials compared with neutral trials in bilateral occipital cortex (Right: 48, - 66, 0; Left: $-44,-66$, 
2). This cluster of activation extended through posterior temporal gyri. A significant interaction effect of group and distractor type was found during the maintenance period. In regions where emotional stimuli elicited greater activation compared to neutral stimuli, healthy control participants elicited significantly greater differences in activation than adults with schizophrenia in bilateral posterior cingulate, right parietal cortex, and bilateral orbital frontal cortex (Figure 3). The MNI coordinates for these activations are provided in Table 3. In posterior cingulate and orbital frontal cortex healthy adults elicited a larger hemodynamic response to emotional trials compared to neutral trials, but in patients this region did not significantly respond to either trial type. In parietal cortex both groups elicited a significant hemodynamic response to both trial types; however, only in healthy controls was the magnitude of the response differentiated by trial type. There were no regions in which the patient group elicited greater activation than healthy controls.

For those regions in which there was a significant interaction of group and trial type, we also conducted anatomically based region of interest (ROI) analyses. We conducted one additional anatomical ROI analysis based on an a priori region of interest: the amygdala. For each region, we conducted an ANOVA with three factors: group (patient, healthy control), trial type (emotional, neutral), and hemisphere (right, left). In bilateral orbital frontal cortex $(\mathrm{OFC})$, bilateral posterior cingulate (PC), bilateral amygdala, and right parietal cortex there were significant main effects of trial type (OFC: $\mathrm{F}=71.66$ (1), $\mathrm{p}<.001$; $\mathrm{PC}: \mathrm{F}=63.3$ (1), $\mathrm{p}<$. 001; Amygdala: $\mathrm{F}=48.8, \mathrm{p}<.001$; R Parietal: $\mathrm{F}=19.06$ (1), $\mathrm{p}<.001)$ and significant interactions of group and trial type (OFC: $\mathrm{F}=113.56$ (1), $\mathrm{p}<.001$; $\mathrm{PC}: \mathrm{F}=187.13, \mathrm{p}<.001$; Amygdala: $\mathrm{F}=22.65$ (1), $\mathrm{p}<.001$; R Parietal: $\mathrm{F}=71.75$ (1), $\mathrm{p}<.001$ ). Although overall, emotional trials elicited a larger response than neutral trials, this pattern was seen in healthy controls but not patients. Thus the differentiation to emotional and neutral trials seen in healthy controls may be the driving factor behind the main effect of trial type. Additionally, in bilateral posterior cingulate there was a significant main effect of group $(\mathrm{F}=9.85, \mathrm{p}=.002)$, in which healthy controls overall elicited larger hemodynamic responses than patients.

Probe (Retrieval) Period-The primary comparisons examined the difference between emotional and neutral trials for each participant group, and the interaction of trial type and participant group. Healthy young adults elicited greater activation to emotional trials compared to neutral trials in bilateral posterior cingulate and precuneus (Right: $8,-52,30$; Left: $-8,-58,30$ ), bilateral orbital and medial frontal gyri (Right: 6, 38, -14; Left: $-10,52$, -2 ) and bilateral middle and superior temporal gyri (Right: 50, -34, 4; Left: $-56,-20,-2$ ). Individuals with schizophrenia did not differentiate emotional and neutral trials during the probe period. During the probe period there was a significant interaction of group and trial type in bilateral posterior cingulate (Centroid: 0, -44, 26; Max Z-value: 3.11, 561 Voxels), see Figure 4. Emotional trials elicited a larger response than neutral trials, but only in the healthy control group. See Figure 3 for hemodynamic responses during the probe period.

Again, we conducted anatomical ROI analyses for the region in which there was a significant interaction of group and trial type, posterior cingulate. A $2 \times 2 \times 2$ ANOVA was conducted with group, trial type, and hemisphere as the factors. There were significant main effects of group (healthy control > patient; $\mathrm{F}=10.23$ (1), $\mathrm{p}<.001$ ) and trial type (emotional > neutral; $F=105.91$ (1), $\mathrm{p}<.001)$. In addition there was a significant interaction of group and trial type $(\mathrm{F}=85.77(1), \mathrm{p}<.001)$, where healthy control participants elicited a greater response to emotional trials compared with neutral trials, and patients did not differentiate these trial types. These results may reflect the continued influence of the emotional distractors on the healthy control group's pattern of activation. Because there was a significant effect of age across groups it is possible that the fMRI effects that we observed during the maintenance and probe periods were due to an effect of age. To assess this possibility we conducted a separate analysis including age as a covariate. We did not 
observe a significant main effect of age or significant differences in the effect of age between the two groups.

Secondary Task-There were no significant differences in activation during any of the task periods when we examined the influence of the secondary task (face detection).

\section{DISCUSSION}

In the current study, we used functional Magnetic Resonance Imaging (fMRI) to examine the influence of emotion on distraction during a verbal working memory task. In each trial, healthy adults and individuals with schizophrenia were asked to remember eight words. The memoranda were followed by distractor pictures that were either neutral or negative in valence. Then each participant viewed pairs of words, and decided which word was part of the initial memoranda. As anticipated, no significant differences in brain activation were found between the trial types during the encode period; this was expected as the trial type was indistinguishable during this phase. There were also no significant differences between subject groups during the encode period, suggesting that both groups processed the memoranda similarly. However, during the maintenance and probe periods, the voxel-based and region of interest fMRI results showed that there was a significant interaction of group and distractor type. During the maintenance period, healthy adults elicited greater activation to emotional distractors compared with neutral distractors in bilateral orbital frontal cortex, bilateral cingulate cortex, and right parietal cortex. This effect persisted in bilateral posterior cingulate during the probe periods. Differences in activation across trial types were not seen in the patient group, resulting in a significant difference in how the groups processed emotional and neutral trials. Additional region of interest analyses revealed a significant interaction of group and distractor type in bilateral amygdala during the maintenance period, such that emotional trials elicited greater activation than neutral trials in the healthy controls but not in the patients.

The regions that differentiated emotional and neutral trials between the groups are consistent with prior research on emotional processing and memory. Engagement of orbital frontal cortex and the amygdala during processing of emotional distractors is consistent with previous fMRI research that supports involvement of ventral frontal brain regions and the amygdala in response to emotional stimuli (Dolcos et al., 2008; Dolcos, Kragel, Wang, \& McCarthy, 2006a; Dolcos et al., 2006b; Phelps et al., 2005; Wang et al., 2006; Wang et al., 2005; Yamaskai et al., 2002). Similarly, ventral frontal brain regions have also been found to be sensitive to anticipation of negative outcomes (Ursu, Clark, Stenger, \& Carter, 2008), and reward outcomes (Knutson, Fong, Adams, Hommer, \& Varner, 2001). Additional research on interoception and emotion has indicated involvement of anterior insular cortex in these processes (Craig, 2002; 2009), however we did not observe activation in this region in the present study.

Two other regions that differentiated emotional and neutral trials in healthy controls, but not patients included posterior cingulate and parietal cortex. Previous fMRI research has suggested that posterior cingulate cortex has a role in episodic memory (Maddock, Garrett, $\&$ Buonocore, 2001). Moreover, others have suggested that this region may be sensitive to both emotion and memory. For example, activation within this region was found when participants evaluated positive and negative words, but not neutral words (Maddock, Garrett, $\&$ Buonocore, 2003). Posterior cingulate and parietal cortex have also been reported as primary brain regions in the default network (Greicius, Krasnow, Reiss, \& Menon, 2003; Raichle et al., 2001). Several studies have suggested that the functional connectivity found among default network regions is weaker in adults with schizophrenia (Bluhm et al., 2007; Calhoun, Maciejewski, Pearlson, \& Kiehl, 2008; Garrity et al., 2007; Liang et al., 2006; 
Williamson, 2007). For example, Bluhm and colleagues found that low frequency oscillations between posterior cingulate and parietal, frontal, and cerebellar regions were less correlated in patients compared to controls (Bluhm et al., 2007). Others have reported spatial differences in the default network regions among individuals with schizophrenia (Garrity et al., 2007). Moreover differences in default network connectivity may be related to positive and negative symptom severity (Bluhm et al., 2007; Garrity et al., 2007).

More recent research examining functional connectivity has proposed that the brain consists of cortical hubs (Buckner et al., 2009). These hubs, present largely in association cortices, act as regions in which information from spatially distant and functionally distinct regions can be integrated. Of relevance to the present study, two regions that were highly interconnected included posterior cingulate and parietal cortex. These core hubs are similar to regions that have been implicated in the default network; however, recent work by Buckner and colleagues demonstrated that these networks were functionally connected both during an active task and during rest. This finding is of particular relevance to the present study, as involvement of parietal cortex and the posterior cingulate occurred during the maintenance and probe periods of a task.

Our findings also highlight an overall reduced hemodynamic response in patients compared to healthy controls. As noted before, patients elicited less activation to emotional stimuli compared to healthy controls. In addition, there were no regions during any phase of the task, in which patients elicited more activation than healthy controls, and healthy controls elicited greater activation overall during the probe period. The decreased activation we observed for patients is consistent with some prior research. For example, several investigators have reported decreased activation in prefrontal cortex during an N-back task in individuals with schizophrenia compared to controls (Barch et al., 2001; Pae et al., 2008; Perlstein et al., 2003). Others have reported similar levels of activation between patients and healthy adults when task difficulty is low, but less activation in patients as difficulty increased (Perlstein et al., 2001). However, in contrast to these findings, other studies have reported increased activation in prefrontal regions during a working memory task for patients compared to controls, which the authors attributed to cortical inefficiency (Potkin et al., 2009; Schneider et al., 2007; van Raalten et al., 2008). The conceptualization of cortical inefficiency in schizophrenia is not inconsistent with the results of the present study, although clearly the direction of change is different (i.e., less activation vs. more activation). Furthermore, this characterization of activation as being inefficient is consistent with reports of dissociations between the magnitude of activation and performance measures among patients. For example, Honey and colleagues noted that levels of activation in parietal cortex correlated with behavioral measures in healthy controls, but not in patients (Honey et al., 2002).

One important caveat to the present results is that the two groups differed in several ways. First, there were more healthy adults than patients in the present study (17 vs. 11). Although we observed significant patterns of activation among the patients, having a smaller number in this group may have resulted in an underestimation in their overall activity. Second, there were differences in the numbers of completed runs across participants. However, on average, the groups completed similar numbers of runs (average number of runs for patients $=6.91$ runs, controls $=6.35$ runs). Moreover the FSL data analysis package that we used takes into account run, session, and participant variance in calculating group-level statistics. Third, patients were significantly older and more variable in age than the healthy controls. However, we did not observe a significant main effect of age in our fMRI data analyses or a significant difference in the effect of age on functional activation between the groups. One final difference between the groups was significant group differences in task response times and accuracies. However, importantly, there was not a significant interaction between trial 
type and group in either measure. While performance differences may have influenced overall patterns of brain activation, they should not have differentially influenced functional activations by trial type, which was our primary comparison of interest. One comparison of interest that we were unable to examine because of the blocked task design was a comparison of activation to correct and incorrect trials.

The present study examined the role of emotional distractors in a verbal working memory task in healthy adults and individuals with schizophrenia. Healthy adults but not patients differentiated emotional and neutral distractor images during the maintenance period in bilateral orbital frontal cortex, bilateral posterior cingulate, and right parietal cortex. This group by trial type interaction persisted during the probe period in posterior cingulate. These results suggest that individuals with schizophrenia fail to differentiate emotional and neutral events and that emotional and neutral distracters have a similar effect on executive function and performance in schizophrenia.

\section{Acknowledgments}

This work was supported in part by the NIH through the following NCRR grant: the Biomedical Informatics Research Network (1 U24 RR025736-01).

\section{References}

Anticevic A, Repovs G, Barch DM. Resisting emotional interference: brain regions facilitating working memory performance during negative distraction. Cognitive, Affective, and Behavioral Neuroscience. 2010; 10:159-73.

Barch DM, Carter CS, Braver TS, Saab FW, MacDonald A, Noll DC, Cohen JD. Selective deficits in prefrontal cortex function in medication-naive patients with schizophrenia. Archives of General Psychiatry. 2001; 58:280-288. [PubMed: 11231835]

Beckman CF, Jenkinson M, Smith SM. General multi-level linear modeling for group analysis in FMRI. Neuroimage. 2003; 20:1052-1063. [PubMed: 14568475]

Bluhm RL, Miller J, Lanius RA, Osuch EA, Boksman K, Neufeld RW, Théberge J, Schaefer B, Williamson P. Spontaneous low-frequency fluctuations in the BOLD signal in schizophrenic patients: anomalies in the default network. Schizophrenia Bulletin. 2007; 33:1004-12. [PubMed: 17556752]

Buckner RL, Sepulcre J, Talukdar T, Krienen FM, Liu H, Hedden T, Andrews-Hanna JR, Sperling RA, Johnson KA. Cortical hubs revealed by intrinsic functional connectivity: Mapping, assessment of stability, and relation to Alzheimer's disease. Journal of Neuroscience. 2009; 29:1860-1873. [PubMed: 19211893]

Calhoun VD, Maciejewski PK, Pearlson GD, Kiehl KA. Temporal lobe and "default" hemodynamic brain modes discriminate between schizophrenia and bipolar disorder. Human Brain Mapping. 2008; 29:1265-1275. [PubMed: 17894392]

Clapp WC, Rubens MT, Gazzaley A. Mechanisms of working memory disruption by external interference. Cerebral Cortex. 2010; 20:859-72. [PubMed: 19648173]

Cohen JD, Forman SD, Braver TS, Casey BJ, Servan-Schreiber D, Noll DC. Activation of prefrontal cortex in a nonspatial working memory task with functional MRI. Human Brain Mapping. 1994; 1:293-304.

Cohen JD, Perlstein WM, Braver TS, Nystrom LE, Noll DC, Jonides J. Temporal dynamics of brain activation during a working memory task. Nature. 1997; 386:604-608. [PubMed: 9121583]

Coltheart M. The MRC psycholinguistic database. Quarterly Journal of Experimental Psychology: Human Experimental Psychology. 1981; 33A:497-505.

Conklin HM, Curtis CE, Katsanis J, Iacono WG. Verbal Working Memory Impairment in Schizophrenia Patients and Their First-Degree Relatives: Evidence From the Digit Span Task. American Journal of Psychiatry. 2000; 157:275-277. [PubMed: 10671401] 
Copland DA, De Zubicaray GI, McMahon KL, Eastburn MM. Neural correlates of semantic priming for ambiguous words: An event-related fMRI study. Brain Research. 2007; 1131:163-172. [PubMed: 17173868]

Craig AD. How do you feel? Interoception: the sense of the physiological condition of the body. Nature Reviews Neuroscience. 2002; 3:655-666.

Craig AD. How do you feel - now? The anterior insula and human awareness. Nature Reviews Neuroscience. 2009; 10:59-70.

Demb JB, Desmond JE, Wagner AD, Vaidya CJ, Glover GH, Gabrieli JDE. Semantic encoding and retrieval in the left inferior prefrontal cortex: a functional MRI study of task difficulty and process specificity. Journal of Neuroscience. 1995; 15:5870-5878. [PubMed: 7666172]

Demonet JE, Chollet F, Ramsay S, Cardebat D, Nespoulous JL, Wise RSJ, Rascal A, Frackowiak RSJ. The anatomy of phonological and semantic processing in normal subjects. Brain. 1992; 115:17531768. [PubMed: 1486459]

Dichter GS, Bellion C, Casp M, Belger A. Impaired modulation of attention and emotion in schizophrenia. Schizophrenia Bulletin. 2009; 36:595-606. [PubMed: 18843096]

Dolcos F, Diaz-Granados P, Wang L, McCarthy G. Opposing influences of emotional and nonemotional distracters upon sustained prefrontal cortex activity during a delayed-response working memory task. Neuropsychologia. 2008; 46:326-335. [PubMed: 17765933]

Dolcos F, Kragel P, Wang L, McCarthy G. Role of the inferior frontal cortex in coping with distracting emotions. Neuroreport. 2006a; 17:1591-1594. [PubMed: 17001274]

Dolcos F, McCarthy G. Brain systems mediating cognitive interference by emotional distraction. Journal of Neuroscience. 2006b; 26:2072-2079. [PubMed: 16481440]

Fichtenholtz HM, Dean HL, Dillon DG, Yamasaki H, McCarthy G, LaBar KS. Emotion-attention network interactions during a visual oddball task. Cognitive Brain Research. 2004; 20:67-80. [PubMed: 15130591]

Gadde, S.; McCarthy, G. 2009. http://www.birncommunity.org/tools-catalog/bxhxcedetools/

Garrity AG, Pearlson GD, McKiernan K, Lloyd D, Kiehl KA, Calhoun VD. Aberrant "Default Mode" Functional Connectivity in Schizophrenia. American Journal of Psychiatry. 2007; 164:450-457. [PubMed: 17329470]

Gold BT, Balota DA, Jones SJ, Powell DK, Smith CD, Andersen AH. Dissociation of automatic and strategic lexical-semantics: Functional magnetic resonance imaging evidence for differing roles of multiple frontotemporal regions. Journal of Neuroscience. 2006; 26:6523-6532. [PubMed: 16775140]

Goldman-Rakic P. Working memory dysfunction in schizophrenia. The Journal of Neuropsychiatry and Clinical Neurosciences. 1994; 6:348-357. [PubMed: 7841806]

Greicius MD, Krasnow B, Reiss AL, Menon V. Functional connectivity in the resting brain: A network analysis of the default mode hypothesis. Proceedings of the National Academy of Sciences. 2003; 100:253-258.

Heinrichs RW, Zakzanis KK. Neurocognitive deficit in schizophrenia: A quantitative review of the evidence. Neuropsychology. 1998; 12:426-445. [PubMed: 9673998]

Honey GD, Bullmore ET, Sharma T. De-coupling of cognitive performance and cerebral functional response during working memory in schizophrenia. Schizophrenia Research. 2002; 53:45-56. [PubMed: 11728837]

Jha AP, McCarthy G. The influence of memory load upon delay-interval activity in a working-memory task: An event-related functional MRI study. Journal of Cognitive Neuroscience. 2000; 12:90-105. [PubMed: 11506650]

Kapur S, Craik FIM, Tulving E, Wilson AA, Houle S, Brown GM. Neuroanatomical correlates of encoding in episodic memory: levels of processing effect. Proceedings of the National Academy of Sciences. 1994; 91:2008-2011.

Keshavan MS, Diwadkar VA, Spencer SM, Harenski KA, Luna B, Sweeney JA. A preliminary functional magnetic resonance imaging study in offspring of schizophrenic parents. Progress in Neuro-psychopharmacology and Biological Psychiatry. 2002; 26:1143-1149. [PubMed: 12452537] 
Kindermann SS, Brown GG, Zorrilla LE, Olsen RK, Jeste DV. Spatial working memory among middle-aged and older patients with schizophrenia and volunteers using fMRI. Schizophrenia Research. 2004; 68:203-216. [PubMed: 15099603]

Knutson B, Fong GW, Adams CM, Hommer D, Varner JL. Dissociation of reward anticipation and outcome with event-related fMRI. Neuroreport. 2001; 12:3683-3687. [PubMed: 11726774]

Kucera, H.; Francis, W. Computational analysis of present-day American English. Providence, RI: Brown University Press; 1967.

Lancaster JL, Woldorff MG, Parsons LM, Liotti M, Freitas CS, Rainey L, Kochunov PV, Nickerson D, Mikiten SA, Fox PT. Automated Talairach Atlas labels for functional brain mapping. Human Brain Mapping. 2000; 10:120-131. [PubMed: 10912591]

Lang, PJ.; Bradley, MM.; Cuthbert, BN. Technical Report A-8. University of Florida; Gainesville, FL: 2008. International affective picture system (IAPS): Affective ratings of pictures and instruction manual.

Liang M, Zhou Y, Liu Z, Tian L, Liu H, Hao Y. Widespread functional disconnectivity in schizophrenia with resting-state functional magnetic resonance imaging. Neuroreport. 2006; 17:209-213. [PubMed: 16407773]

Maddock RJ, Garrett AS, Buonocore MH. Remembering familiar people: the posterior cingulate cortex and autobiographical memory retrieval. Journal of Neuroscience. 2001; 104:667-676.

Maddock RJ, Garrett AS, Buonocore MH. Posterior cingulate cortex activation by emotional words: fMRI evidence from a valence decision task. Human Brain Mapping. 2003; 18:30-41. [PubMed: 12454910]

Manoach DS, Gollub RL, Benson ES, Searl MM, Goff DC, Halpern E, Saper CB, Rauch SL. Schizophrenic subjects show aberrant fMRI activation of dorsolateral prefrontal cortex and basal ganglia during working memory performance. Biological Psychiatry. 2000; 48:99-109. [PubMed: 10903406]

Manoach DS, Press DZ, Thangaraj V, Searl MM, Goff DC, Halpern E, Saper CB, Warach S. Schizophrenic subjects activate dorsolateral prefrontal cortex during a working memory task, as measured by fMRI. Biological Psychiatry. 1999; 45:1128-1137. [PubMed: 10331104]

McCarthy G, Blamire AM, Puce A, Nobre AC, Bloch G, Hyder F, Goldman-Rakic P, Shulman RG. Functional MRI of human prefrontal cortex activation during a spatial working memory task. Proceedings of the National Academy of Sciences. 1994; 91:8690-8694.

Noppenney U, Phillips J, Price CJ. The neural areas that control the retrieval and selection of semantics. Neuropsychologia. 2004; 42:1269-1280. [PubMed: 15178178]

Owen WJ, Borowsky R, Sarty GE. FMRI of two measures of phonological processing in visual word recognition: Ecological validity matters. Brain and Language. 2004; 90:40-46. [PubMed: 15172523]

Pae CU, Juh R, Yoo SS, Choi BG, Lim HK, Lee C, Paik IH, Jeun SS, Lee CU. Verbal working memory dysfunction in schizophrenia: An fMRI investigation. International Journal of Neuroscience. 2008; 118:1467-1487. [PubMed: 18788030]

Paulesu E, Frith CD, Frackowiak RSJ. The neural correlates of the verbal component of working memory. Nature. 1993; 362:342-45. [PubMed: 8455719]

Perlstein WM, Carter CS, Noll DC, Cohen JD. Relation of Prefrontal Cortex Dysfunction to Working Memory and Symptoms in Schizophrenia. American Journal of Psychiatry. 2001; 158:1105-1113. [PubMed: 11431233]

Perlstein WM, Dixit NK, Carter CS, Noll DC, Cohen JD. Prefrontal cortex dysfunction mediates deficits in working memory and prepotent responding in schizophrenia. Biological Psychiatry. 2003; 53:25-38. [PubMed: 12513942]

Petrides ME, Alivisatos B, Meyer E, Evans AC. Functional activation of the human frontal cortex during the performance of verbal working memory tasks. Proceedings of the National Academy of Sciences. 1993; 90:878-882.

Phan KL, Wager T, Taylor SF, Liberzon I. Functional neuroanatomy of emotion: a meta-analysis of emotion activation studies in PET and fMRI. Neuroimage. 2002; 16:331-348. [PubMed: 12030820] 
Phan KL, Wager TD, Taylor SF, Liberzon I. Functional neuroimaging studies of human emotions. CNS Spectrums. 2004; 9:258-266. [PubMed: 15048050]

Phelps EA, Ledoux JE. Contributions of the amygdala to emotion processing: From animal models to human behavior. Neuron. 2005; 48:175-187. [PubMed: 16242399]

Potkin SG, Turner JA, Brown GG, McCarthy G, Greve DN, Glover GH, Manoach DS, Belger A, Diaz M, Wible CG, Ford JM, Mathalon DH, Gollub R, Lauriello J, O’Leary D, van Erp TGM, Toga AW, Preda A, Lim KO. Working memory and DLPFC inefficiency in schizophrenia: The FBIRN study. Schizophrenia Bulletin. 2009; 35:19-31. [PubMed: 19042912]

Raichle ME, MacLeod AM, Snyder AZ, Powers WJ, Gusnard DA, Shulman RG. A default mode of brain function. Proceedings of the National Academy of Sciences. 2001; 98:676-682.

Rissman J, Gazzaley A, D'Esposito M. The effect of non-visual working memory load on top-down modulation of visual processing. Neuropsychologia. 2009; 47:1637-1646. [PubMed: 19397858]

Roskies AL, Fiez JA, Balota DA, Raichle ME, Petersen SE. Taskdependent modulation of regions in the left inferior frontal cortex during semantic processing. Journal of Cognitive Neuroscience. 2001; 13:829-843. [PubMed: 11564326]

Schneider F, Habel U, Reske M, Kellermann T, Stocker T, Shah NJ, Zilles K, Braus DF, Schmitt A, Schlosser R, Wagner M, Frommann I, Kircher T, Rapp A, Meisenzahl E, Ufer S, Ruhrmann S, Thienel R, Sauer H, Henn FA, Gaebel W. Neural correlates of working memory dysfunction in first-episode schizophrenia patients: An fMRI multi-center study. Schizophrenia Research. 2007; 89:198-210. [PubMed: 17010573]

Smith SM. Fast robust automated brain extraction. Human Brain Mapping. 2002; 3:143-155. [PubMed: 12391568]

Smith SM, Jenkinson M, Woolrich MW, Beckman CF, Behrens TEJ, Johansen-Berg H, Bannister PR, De Luca M, Drobnjak I, Flitney DE, Niazy R, Saunders J, Vickers J, Zhang Y, De Stefano N, Brady JM, Matthews PM. Advances in functional and structural MR image analysis and implementation as FSL. Neuroimage. 2004; 23:208-219.

Sweet LH, Paskavitz JF, Haley AP, Gunstad JJ, Mulligan RC, Nyalakanti PK, Cohen RA. Imaging phonological similarity effects on verbal working memory. Neuropsychologia. 2008; 46:11141123. [PubMed: 18155074]

Talairach, J.; Tournoux, P. Co-planar stereotaxic atlas of the human brain. New York: Thieme; 1988.

Thermenos HW, Seidman LJ, Breiter H, Goldstein JM, Goodman JM, Poldrack R, Faraone SV, Tsuang MT. Functional magnetic resonance imaging during auditory verbal working memory in nonpsychotic relatives of persons with schizophrenia: A pilot study. Biological Psychiatry. 2004; 55:490-500. [PubMed: 15023577]

Ursu S, Clark KA, Stenger VA, Carter CS. Distinguishing expected negative outcomes from preparatory control in the human orbitofrontal cortex. Brain Research. 2008; 1227:110-119. [PubMed: 18602375]

van Raalten TR, Ramsey NF, Jansma JM, Jager G, Kahn RS. Automatization and working memory capacity in schizophrenia. Schizophrenia Research. 2008; 100:161-171. [PubMed: 18155446]

Voyvodic JT. Real-time fMRI integrating paradigm control, physiology, behavior and on-line statistical analysis. Neuroimage. 1999; 10:91-106. [PubMed: 10417244]

Wang L, Labar KS, McCarthy G. Mood alters amygdala activation to sad distractors during an attentional task. Biological Psychiatry. 2006; 60:1139-1146. [PubMed: 16713587]

Wang L, McCarthy G, Song AW, Labar KS. Amygdala activation to sad pictures during high-field (4T) functional magnetic resonance imaging. Emotion. 2005; 5:12-22. [PubMed: 15755216]

Williamson P. Are Anticorrelated Networks in the Brain Relevant to Schizophrenia? Schizophrenia Bulletin. 2007; 33:994-1003. [PubMed: 17493957]

Wilson MD. The MRC Psycholinguistic Database: Machine Readable Dictionary, Version 2. Behavioural Research Methods, Instruments and Computers. 1988; 20:6-10.

Woolrich MW, Behrens TEJ, Beckman CF, Jenkinson M, Smith SM. Multi-level linear modelling for FMRI group analysis using Bayesian inference. Neuroimage. 2004; 21:1732-1747. [PubMed: 15050594] 
Woolrich MW, Jbabdi S, Patenaude B, Chappell M, Makni S, Behrens TEJ, Beckman CF, Jenkinson M, Smith SM. Bayesian analysis of neuroimaging data in FSL. Neuroimage. 2009; 45:S173-186. [PubMed: 19059349]

Woolrich MW, Ripley BD, Brady JM, Smith SM. Temporal autocorrelation in univariate linear modelling of FMRI data. Neuroimage. 2001; 6:1370-1386. [PubMed: 11707093]

Yamaskai H, Labar KS, McCarthy G. Dissociable prefrontal brain systems for attention and emotion. Proceedings of the National Academy of Sciences. 2002; 99:11447-11451.

Zatorre RJ, Evans AC, Meyer E, Gjedde A. Lateralization of phonetic and pitch discrimination in speech processing. Science. 1996; 256:846-849. [PubMed: 1589767] 


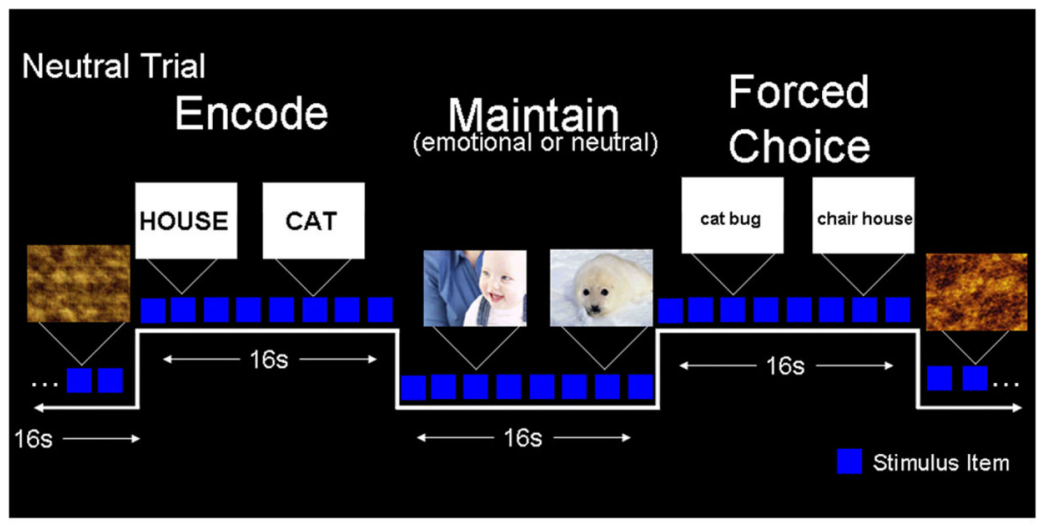

Figure 1.

Experimental Task.

An example of a neutral trial is shown. Each trial consisted of three phases: encoding word memoranda, maintaining the memoranda while viewing distractor photographs, and a forced-choice probe period to assess memory. Emotional and neutral trials were identical in format except for the valence of the distracter photographs. 


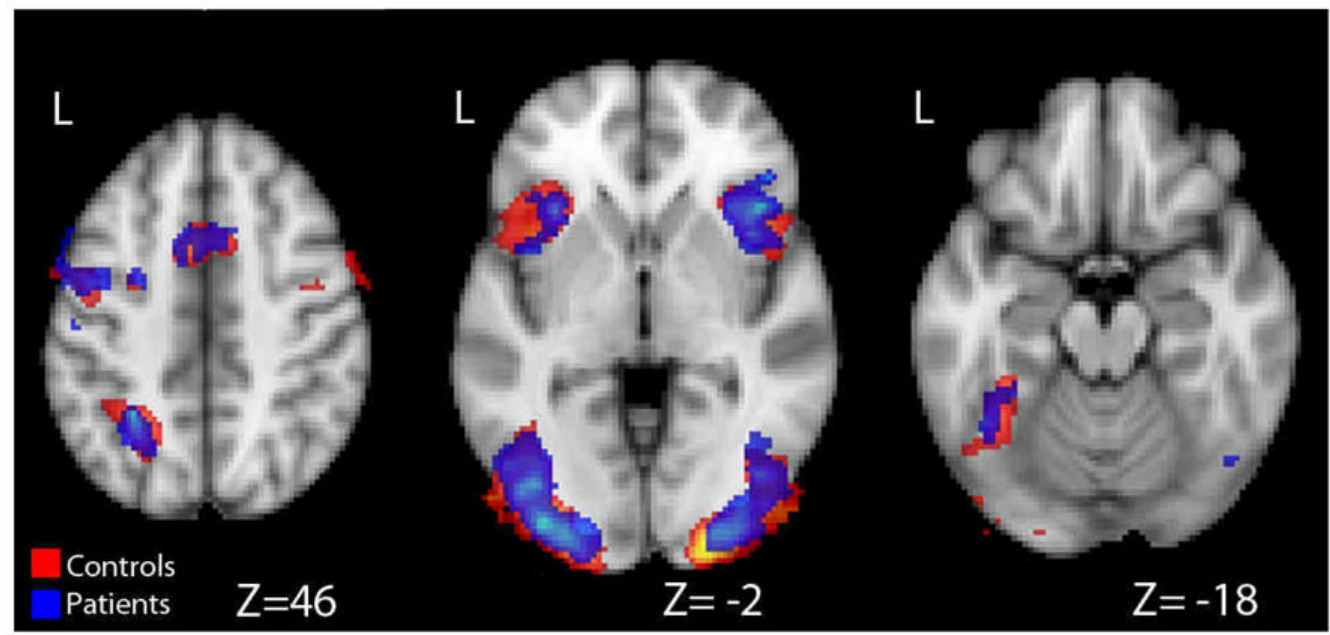

Figure 2.

Activation During the Encode Period.

Overall activation to emotional and neutral trials during the encode period is displayed. There were no significant differences between groups or between trial types within groups. Largely overlapping patterns of activation for healthy controls (red) and patients (blue) can be seen. 

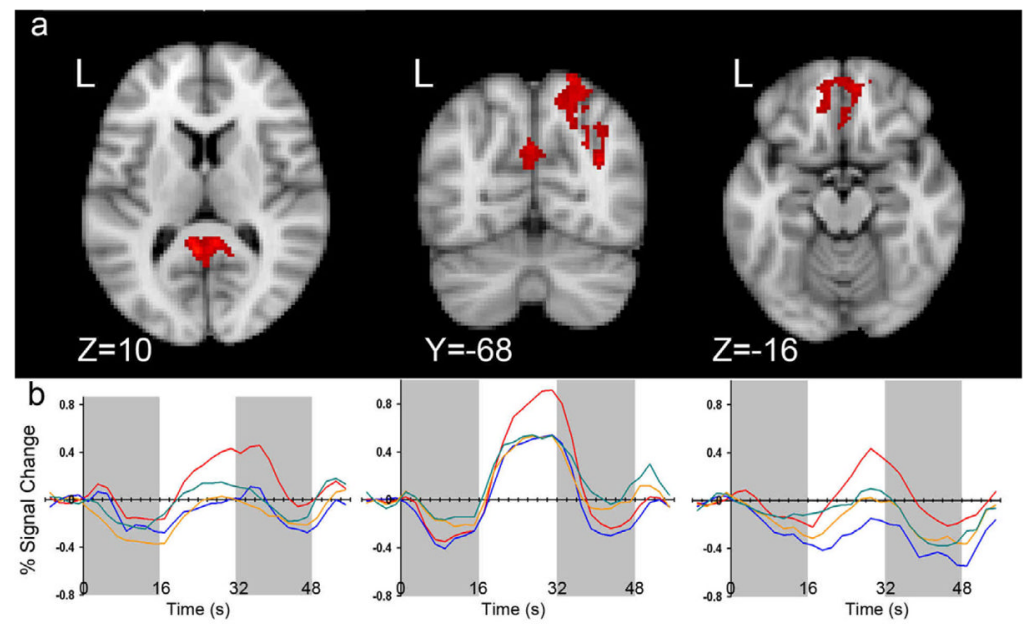

Figure 3.

Activation During the Maintenance Period.

a. Areas in red indicate regions where the difference between emotional and neutral trials (emotional > neutral) was greater for healthy control participants compared to schizophrenic patients. These differences can be seen in three regions: bilateral posterior cingulate, right parietal cortex, and bilateral orbital frontal cortex. b. The corresponding hemodynamic responses (HDRs) for emotional (red) and neutral (blue) trials for healthy controls and schizophrenic patients (emotional = orange, neutral $=$ green $)$ are shown. HDRs shown are for the entire trial. Encode, maintenance, and probe periods are denoted by the gray and white bars in the background. 


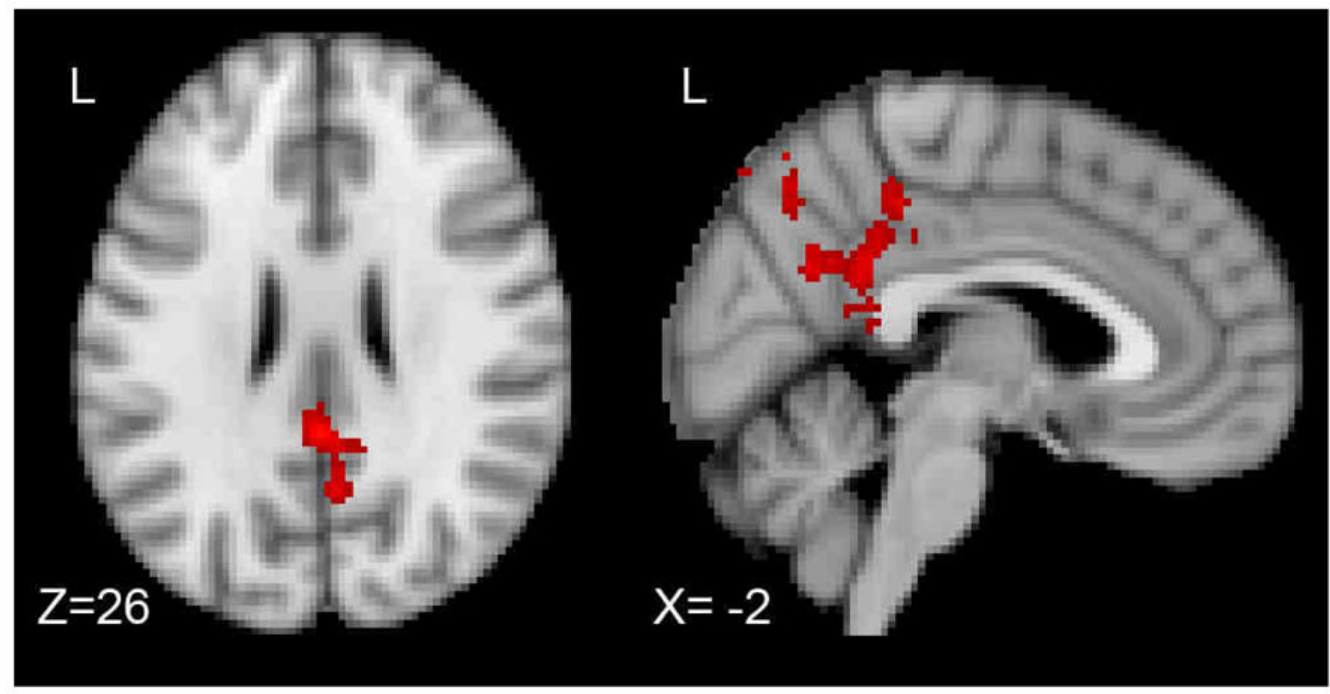

Figure 4.

Activation During the Forced Choice Probe Period.

Areas in red indicate regions where healthy controls elicited a significantly greater difference between emotional and neutral trials (emotional > neutral) compared to schizophrenic patients. These differences can be seen in bilateral posterior cingulate. 


\section{Table 1}

Demographic and Clinical Characteristics for Patients

\begin{tabular}{|c|c|c|}
\hline & Patients & Healthy Controls \\
\hline Age $*$ & $32.57 \pm 12.7(20-59)$ & $24.01 \pm 3.89(19-30)$ \\
\hline \multicolumn{3}{|l|}{ Ethnic Groups $* * *$} \\
\hline White/Caucasian (n) & $81.82 \%(9)$ & $64.71 \%(11)$ \\
\hline African American (n) & $18.18 \%(2)$ & $29.41 \%(5)$ \\
\hline Multi-racial (n) & $0 \%(0)$ & $5.88 \%(1)$ \\
\hline \multicolumn{3}{|l|}{ Gender $* * *$} \\
\hline Male (n) & $90.91 \%(10)$ & $41.17 \%(7)$ \\
\hline Female (n) & $9.09 \%(1)$ & $58.83 \%(10)$ \\
\hline PANSS $^{* *}$ Total & $66.40 \pm 15.33(42-87)$ & \\
\hline Positive Total & $15 \pm 4.22(8-20)$ & \\
\hline Negative Total & $18 \pm 6.15(9-25)$ & \\
\hline General Total & $33.40 \pm 6.57(23-42)$ & \\
\hline \multicolumn{3}{|l|}{ Parental Education $*$} \\
\hline Mother & $12.73 \pm 3.61(4-18)$ & \\
\hline Father & $13.40 \pm 4.33(4-18)$ & \\
\hline \multicolumn{3}{|c|}{ Data are given as mean years \pm SD (range) } \\
\hline \multicolumn{3}{|c|}{ ** PANSS, Positive and Negative Syndrome Scale } \\
\hline
\end{tabular}




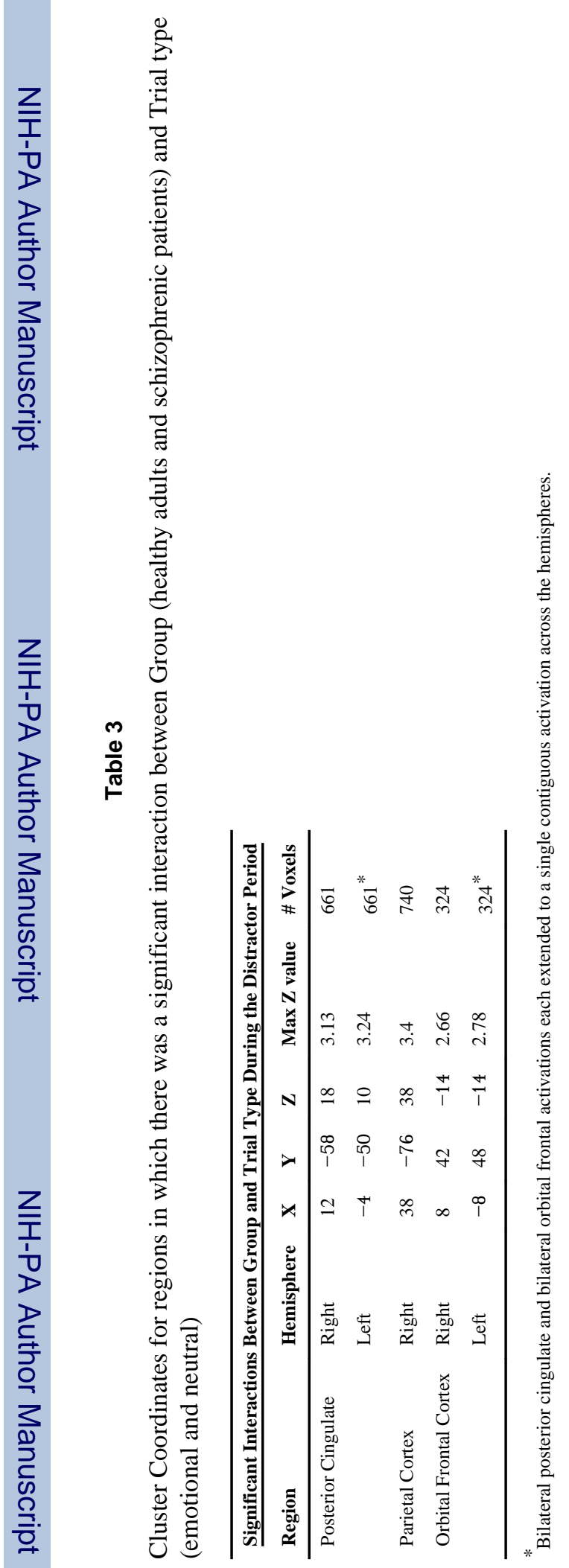

